\title{
ESR Spectrum and Magnetic Behavior of Copper(II) Sebacate
}

L. Lezama ${ }^{\text {a }}$, T. Rojo ${ }^{\text {a }}$, E. J. Baran ${ }^{\text {b }}$, and M.H. Torre ${ }^{\text {c }}$

a Departamento de Química Inorgánica, Facultad de Ciencias, Universidad del País Vasco, E-48080 Bilbao, Spain

b Química Inorgánica (CEQUINOR), Facultad de Ciencias Exactas,

Universidad Nacional de La Plata, C. Correo 962, 1900-La Plata, Argentina

c Química Inorgánica, Facultad de Química, Universidad de la República, Montevideo, Uruguay

\section{Z. Naturforsch. 51 a, 831-833 (1996); received February 14, 1996}

The magnetic susceptibility of copper(II) sebacate, measured between 5 and $400 \mathrm{~K}$, confirms the presence of strongly coupled $\mathrm{Cu}-\mathrm{Cu}$ pairs. The ESR spectrum, obtained with a polycrystalline sample, shows an important rhombic anisotropy and supports a $d_{x^{2}-y^{2}}$ ground-state.

Key words: Copper(II) sebacate; ESR spectrum; Magnetic behavior.

\section{Introduction}

Different attempts have been made to correct the abnormalities related to Menkes' disease, an inherited disorder associated with problems in copper absorption [1-3]. Copper supplementation has attained great interest not only in relation with this and other physiological disorders $[4,5]$ but also in veterinary medicine [6]. The copper(II) complex of sebacic acid (decanedioic acid) appears as a very promising system in this context.

In recent papers we described a method for the facile synthesis of this complex, complemented with spectroscopic studies, solubility tests and lipophilicity assays $[7,8]$.

In order to advance in a more complete characterization of this interesting compound, we have investigated its magnetic behavior through magnetic susceptibility measurements and ESR spectroscopy.

\section{Experimental}

Copper(II) sebacate, $\mathrm{CuC}_{10} \mathrm{H}_{16} \mathrm{O}_{4}$, was obtained by treating sodium sebacate with copper(II) sulfate, following the procedure described in [7]. It was characterized by chemical analysis and infrared spectroscopy $[7,8]$.

Reprint requests to Prof. E. J. Baran.
Magnetic susceptibility measurements were performed between 5 and $400 \mathrm{~K}$ using a Quantum Design SQUID magnetometer employing a magnetic field of $1 \mathrm{kG}$.

The ESR spectra were recorded at 100 and $300 \mathrm{~K}$ with a Bruker-300 spectrometer using $34.01 \mathrm{GHz}$ ( $Q$-band). The frequency of the cavity was determined with a Hewlett-Packard 5352 B microwave frequency counter, and the magnetic fields by means of a Bruker NMR, model ER035 Gaussmeter.

\section{Results and Discussion}

Our previous studies of the complex demonstrate the existence of a dimeric structure containing $\mathrm{Cu}-\mathrm{Cu}$ moieties, bridged by carboxylate groups [7], similar to that found in the well known copper(II) acetate and other $\mathrm{Cu}(\mathrm{II})$ complexes of carboxylic acids [9-11].

The plot of the magnetic susceptibility as a function of temperature, shown in Fig. 1, is characteristic for strongly coupled dimeric copper units. It shows a broad maximum at approximately $260 \mathrm{~K}$ and a clear trend to diminish at lower temperatures, an usual behavior in discrete antiferromagnetically ordered systems. Below $30 \mathrm{~K}$, the susceptibility increases, probably due to the presence of a small paramagnetic impurity.

The observed magnetic behavior can be described by an equation derived by Bleaney and Bowers [12], based on the Heisenberg isotropic spin Hamiltonian, $H=-2 J S_{1} S_{2}$, for two coupled $S=1 / 2$ systems, and introducing an additional term which accounts for the 


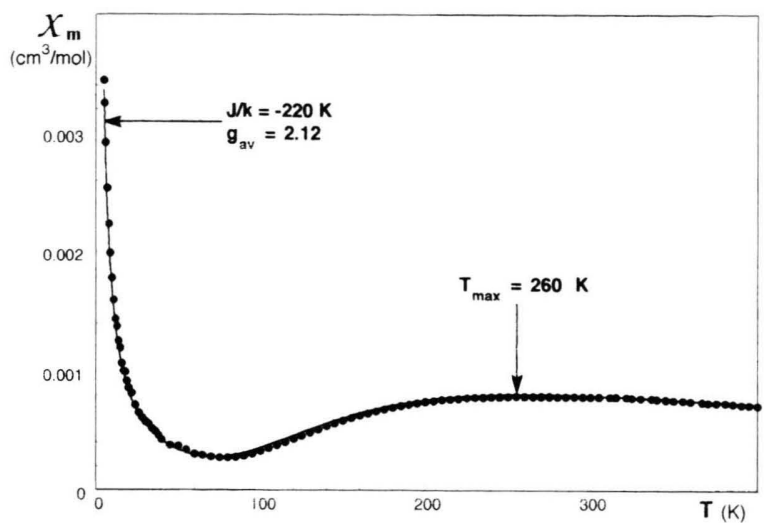

Fig. 1. Dependence of the molar magnetic susceptibility of copper(II) sebacate on temperature.

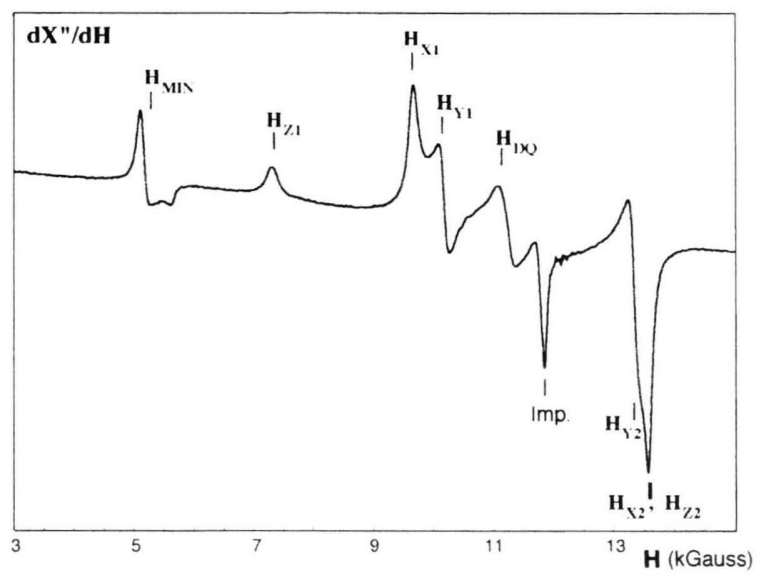

Fig. 2. $Q$-band ESR spectrum of copper(II) sebacate at $100 \mathrm{~K}$.

paramagnetic contribution:

$$
\begin{aligned}
X= & (1-\varrho) \frac{N g^{2} \beta^{2}}{k T}\left[\frac{1}{1+\exp (-2 J / k T)}\right] \\
& +\varrho \frac{N g^{2} \beta^{2}}{4 k T},
\end{aligned}
$$

where all terms have their usual meaning and $\varrho$ is the percentage of the paramagnetic impurity.

The best fit of experimental data is attained with the following parameters:

$J / k=-220 \mathrm{~K}\left(153 \mathrm{~cm}^{-1}\right), \quad g=2.11, \quad \varrho=0.04$.

The value obtained for $J$ is totally reasonable for our system. Taking into account the temperature at which the maximum of the susceptibility is observed, $T\left(\chi_{\max }\right)=1.2 \mathrm{~J} / k=260 \mathrm{~K}, 217 \mathrm{~K}$ is obtained for $\mathrm{J} / k$, confirming the validity of the used model.

The ESR spectrum registered in the $Q$-band and shown in Fig. 2 is characteristic for a triplet $S=1$ state with appreciable zero-field splitting, which probably arises from the combined effects of the anisotropic and antisymmetric exchange and from the classical dipole-dipole interaction. The spectrum could be interpreted with the spin Hamiltonian

$$
H_{S S}=\beta H g S+D\left(S_{z^{2}}-2 / 3\right)+E\left(S_{x^{2}}-S_{y^{2}}\right),
$$

where $D=3 / 2 D_{z}$ and $E=1 / 2\left(D_{x}-D_{y}\right)$ are the parameters related to the zero-field splitting of the triplet state. In a system with rhombic symmetry, and for randomly oriented samples, six spin allowed transitions $\left(\Delta M_{s}= \pm 1\right)$ may be observed if $h v>D>3 E$. The magnetic fields at which these transitions can be observed $\left(H_{\mathrm{X} 1}, H_{\mathrm{X} 2}, H_{\mathrm{Y} 1}, H_{\mathrm{Y} 2}, H_{\mathrm{Z} 1}, H_{\mathrm{Z} 2}\right)$ were determined by Waserman et al. [13], giving six equations depending on the microwave frequency and the eigenvalues of the $g$ and $D$ tensors. This method has the restriction that both tensors have the same principal axes.

Besides these allowed transitions, there are two forbidden ones $\left(\Delta M_{s}= \pm 2\right)$, which are frequently observed [14]. One of them $\left(H_{\mathrm{MIN}}\right)$ corresponds to the absorption of one photon, which induces a transition between the levels $M_{\mathrm{s}}=-1$ and $M_{\mathrm{s}}=+1$. The other one $\left(H_{\mathrm{DQ}}\right)$, corresponds to the absorption of two quanta, which generate the same transition [14].

Therefore one obtains a self-consistent set of eight equations which can be solved by repeated interaction in order to obtain the parameters of interest, namely $g_{x}, g_{y}, g_{z}, D$ and $E$. Adjusting the observed signals by means of a least squares refinement to the respective equations, the following values were obtained:

$$
\begin{aligned}
& g_{x}=2.069, \quad g_{y}=2.043, \quad g_{z}=2.321, \\
& D=0.338 \mathrm{~cm}^{-1}, \quad E=-0.012 \mathrm{~cm}^{-1} .
\end{aligned}
$$

The marks in Fig. 2 indicate the calculated positions of the transition. This analysis does not allow to obtain the real signs of $D$ and $E$ and shows only the relative values of one with respect to the other. Notwithstanding, they are important in the assignment of the observed transitions, specially in cases such as the present one, in which the anisotropy of the $g$ tensor is important.

Although a clear rhombic anisotropy is evident, all the obtained parameters are of the same order as that 
usually observed in classic $\mathrm{Cu}(\mathrm{II})$ dimers [14-16], but the use of the $Q$-band in the measurements shows an evident rhombic anisotropy that is often lost when the experiments were carried out with the $X$-band.

On the other hand, and as the lowest $g$-value is higher than 2.04, one can deduce that $d_{x^{2}-y^{2}}$ must be the ground state of $\mathrm{Cu}(\mathrm{II})$ in this complex. Therefore, the environment of the metal center may be an elongated octahedron or a distorted square based pyramid [17].

The spectrum of Fig. 2 also shows a signal corresponding to an uncoupled $\mathrm{Cu}(\mathrm{II})$ impurity, probably the same which gives rise to the paramagnetic contribution observed at low temperatures in the susceptibility measurements. This signal is probably due to

[1] J. H. Menkes, G. K. Steigleder, D. R. Weakley, and J. H. Sung, Pediatrics 29, 764 (1962).

[2] D. M. Danks, B. J. Stevens, P. E. Campbell, J. M. Gillespie, J. Walker-Smith, J. Blomfield, and B. Turner, Lancet 1, 1100 (1972).

[3] D. M. Danks, P. E. Campbell, B. J. Stevens, V. Mayne, and E. Cartwright, Pediatrics 50, 189 (1972).

[4] B. Sarkar, in: Metal Ions in Biological Systems, Vol. 12. Properties of Copper, Ed. H. Sigel, Marcel Dekker, New York 1981, p. 233, and references therein.

[5] E. J. Baran, Acta Farm. Bonaerense 4, 125 (1985).

[6] M. E. Smart, N. F. Cymbalul, and D. A. Christensen, Can. Veter. J. 33, 163 (1992), and references therein.

[7] E. J. Baran, S. B. Etcheverry, M. H. Torre, and E. Kremer, Polyhedron 13, 1859 (1994).

[8] E. J. Baran, S. B. Etcheverry, M. H. Torre, and E. Kremer, Acta Farm. Bonaerense 13, 85 (1994).

[9] F. A. Cotton and G. Wilkinson, Advanced Inorganic Chemistry, 4th Edit., J. Wiley, New York 1980. the presence of the monomeric species resulting from breaks of the $\mathrm{Cu}-\mathrm{Cu}$ dimers.

To conclude, this study allowed to obtain a good insight into the magnetic behavior of copper(II) sebacate, confirming the existence of a pair of strongly coupled $\mathrm{Cu}-\mathrm{Cu}$ dimers and a significant rhombic distortion of the coordination sphere around the metal centers.

\section{Acknowledgements}

This work was supported by the Consejo Nacional de Investigaciones Científicas y Técnicas de la República Argentina.

[10] J. Catterick and P. Thornton, Adv. Inorg. Chem. 20, 291 (1977).

[11] R. J. Doedens, Prog. Inorg. Chem. 21, 209 (1976).

[12] B. Bleaney and K. D. Bowers, Proc. Roy. Soc. London, Ser. A 214, 451 (1952).

[13] E. Waserman, L. C. Snyder, and W. A. Yager, J. Chem. Phys. 41, 1763 (1964)

[14] A. Bencini and D. Gatteschi, Electron Paramagnetic Resonance of Exchange Coupled Systems, Springer, Berlin 1990.

[15] A. Abragam and B. Bleaney, Electron Paramagnetic Resonance of Transition Ions, Oxford University Press, London 1970.

[16] J. R. Pilbrow, Transition Ion Electron Paramagnetic Resonance, Oxford University Press, New York 1990.

[17] B. J. Hathaway and D. E. Billing, Coord. Chem. Rev. 5, 143 (1970). 